\section{A Clinical Study of Plaque Reduction by Viokase}

by

\author{
Don L. Allen* \\ Richard M. COURTNEY**
}

THE REMOVAL OF DEBRIS AND plaque from the teeth and the prevention of calculus formation have long been recognized as important aspects of preventive dentistry. Recent data reconfirm, both at the experimental and epidemiological levels, the strong relationship between dental plaque and periodontal disease. It is clear that more efficient methods of plaque control for the public at large are needed.

Investigations in vivo ${ }^{1-8}$ and in vitro ${ }^{9-12}$ have demonstrated that amylolytic and proteolytic enzymes can be effective in calculus reduction. However, the reports are few and the investigations have not been designed to measure clinically the amount of plaque reduction in human subjects. Further consideration of this potential aid in oral hygiene is therefore indicated.

This investigation was designed to evaluate the effect of a chewing gum containing Viokase* as compared to that of an identical chewing gum without Viokase. The following aspects were evaluated: 1) oral hygiene status as determined by the accumulation of bacterial plaque or stainable deposit, and 2) irritability of the oral mucous membranes as determined either by visible changes in oral soft tissues or by symptoms related by the subjects.

\section{Materials and Method}

The objectives of the study were discussed in advance with the biostatistician who would subsequently analyze the data. He developed a random pattern by which the subjects would be studied. The random sample was used to divide the subjects into two groups. One group was to use the experimental gum containing Vio-

This investigation was supported by a grant from Philip Morris, Inc. and was conducted at the Dental Research Center, University of North Carolina School of Dentistry, Chapel Hill, North Carolina.

This paper was presented in part at the Joint Meeting of the American and Canadian Academies of Periodontology, Montreal, Quebec, Canada, September 16-19, 1970.

*Professor of Periodontology, University of Florida College of Dentistry, Gainesville, Florida 32601.

**Associate Professor of Oral Pathology, University of Michigan School of Dentistry, Ann Arbor, Michigan 48104.

\footnotetext{
*Viobin Corporation, Monticello, Illinois.
}

kase for three weeks and the control gum without the enzymes for three subsequent weeks. An interval of six weeks in which no gum was prescribed elapsed between the two phases of the study. This was to eliminate any residual enzymatic activity which might occur from the experimental gum.

The other group of subjects started on the control gum and completed the second phase of the study on the experimental gum. A six-weeks interval was provided between the two phases.

The manufacturer provided the sugar-free chewing gum in five-stick packs with ten codes, five for the experimental gum and five for the control gum. A doubleblind protocol was used.

Ninety-one of the 98 subjects who started the experiment finished it. The subjects were not given a dental phophylaxis prior to or during the study. They were given no oral hygiene instructions and were told to have no dental consultation or treatment during the investigation.

Two investigators carried out the study. One investigator evaluated the subjects from the standpoint of whether or not the chewing gum produced any irritability or untoward symptoms. The other investigator evaluated the dental plaque status.

On the initial appointment, the subjects were given a health questionnaire to elicit any serious illnesses, allergies or hypersensitivities, or potential symptoms that might mask or distort the subsequent results. Each questionnaire was reviewed by one investigator in the presence of the subject. Any significant comments were noted. Then, with the aid of a dental assistant and with the subject seated in a regular dental operatory, an examination of the lips and oral cavity was made. Any deviation from what was considered to be clinically normal mucous membrane was recorded on a form.

The subject was then moved to an adjacent dental operatory where the other investigator determined the plaque index for the patient. All patients were instructed in the use of disclosing wafers. They were observed while they used the wafers and were instructed to swish the material around for thirty seconds in the mouth after it had dissolved. The Oral Hygiene Index-simplified was used to grade the amount of plaque. ${ }^{13}$ Uniformly calibrated periodontal probes were used in measuring the stainable plaque on the indicated teeth. The scores were recorded on a standard form.

At the completion of the initial examination, the subjects were returned to the staff person, who gave them written and oral instructions in the use of the chewing gum and provided them with a one-week supply. The subjects were instructed to chew five sticks of gum per 
TABLE 1

Frequency distribution of plaque scores for all patients at the initial examination.

\begin{tabular}{|c|c|c|c|c|c|c|c|c|c|c|c|c|c|c|c|c|c|}
\hline Seque & nce & & 1 & 2 & 3 & 4 & 5 & 6 & 7 & 8 & 9 & 10 & 11 & 12 & 13 & 14 & Total \\
\hline \multirow[b]{2}{*}{ E-P } & $\mathrm{E}$ & 0 & 0 & 1 & 2 & 7 & 7 & 8 & 5 & 7 & 2 & 4 & 0 & 1 & 1 & 0 & 45 \\
\hline & $P$ & 0 & 0 & 0 & 3 & 5 & 12 & 6 & 8 & 4 & 1 & 2 & 1 & 3 & 0 & 0 & 45 \\
\hline \multirow{2}{*}{ P-E } & $\mathbf{P}$ & 0 & 0 & 2 & 1 & 1 & 11 & 12 & 7 & 5 & 5 & 1 & 0 & 0 & 0 & 1 & 46 \\
\hline & $\mathrm{E}$ & 0 & 1 & 2 & 2 & 4 & 9 & 14 & 6 & 5 & 0 & 2 & 0 & 1 & 0 & 0 & 46 \\
\hline
\end{tabular}

$\mathrm{E}=$ Experimental gum.

$\mathbf{P}=$ Placebo.

TABLE 2

Plaque scores at the end of one week of chewing the experimental (E) and the placebo (P) gum

\begin{tabular}{|c|c|c|c|c|c|c|c|c|c|c|c|c|c|c|c|c|}
\hline \multicolumn{3}{|c|}{ Sequence 0} & 1 & 2 & 3 & 4 & 5 & 6 & 7 & 8 & 9 & 10 & 11 & 12 & 13 & Total \\
\hline \multirow[b]{2}{*}{ E-P } & E & 0 & 0 & 2 & 5 & 6 & 11 & 6 & 8 & 1 & 2 & 1 & 0 & 2 & 1 & 45 \\
\hline & $\mathbf{P}$ & 0 & 0 & 4 & 5 & 7 & 8 & 4 & 5 & 2 & 3 & 4 & 1 & 1 & 0 & 45 \\
\hline \multirow[t]{2}{*}{ P-E } & $\mathbf{P}$ & 0 & 0 & 3 & 5 & 7 & 10 & 8 & 0 & 7 & 2 & 4 & 0 & 0 & 0 & 46 \\
\hline & E & 0 & 2 & 2 & 5 & 3 & 10 & 6 & 7 & 8 & 1 & 2 & 0 & 0 & 0 & 46 \\
\hline
\end{tabular}

$\mathrm{E}=$ Experimental gum.

$\mathbf{P}=$ Placebo.

TABLE 3

Plaque scores at the end of three weeks of chewing the experimental (E) and placebo (P) gum.

\begin{tabular}{|c|c|c|c|c|c|c|c|c|c|c|c|c|c|c|c|c|}
\hline \multicolumn{3}{|c|}{ Sequence 0} & 1 & 2 & 3 & 4 & 5 & 6 & 7 & 8 & 9 & 10 & 11 & 12 & 13 & Total \\
\hline \multirow[b]{2}{*}{ E-P } & E & 0 & 0 & 4 & 4 & 8 & 8 & 10 & 0 & 3 & 2 & 2 & 1 & 1 & 1 & 45 \\
\hline & $\mathbf{P}$ & 0 & 0 & 3 & 6 & 4 & 8 & 6 & 2 & 3 & 6 & 3 & 2 & 2 & 0 & 45 \\
\hline \multirow[t]{2}{*}{ P-E } & $\mathbf{P}$ & 0 & 2 & 2 & 5 & 6 & 8 & 9 & 1 & 4 & 3 & 3 & 2 & 0 & 1 & 46 \\
\hline & E & 1 & 2 & 3 & 2 & 9 & 9 & 6 & 9 & 2 & 2 & 1 & 0 & 0 & 0 & 46 \\
\hline
\end{tabular}

$\mathrm{E}=$ Experimental gum.

$\mathbf{P}=$ Placebo.

day, evenly spaced throughout the waking hours. The gum was provided in typical five-stick packs without labels. The subjects were evaluated at the end of one and three weeks.

Since it was conceivable that there might be some continuing effect from the enzymes, an interval of six weeks was provided before the second phase of the study started. The groups were reversed for the second part of the study but followed the same schedule for examinations. The subjects were appointed the same day of the week and the same time of the day for each examination. At the end of the study, the subjects filled out the questionnaire again.

\section{RESULTS}

All of the forms used in the study and the decoding information were given to a biostatistician. Seven people did not complete the study. No pattern of age, sex, or type of gum used could be ascertained in those who failed to complete the study. The subsequent analysis of 91 subjects is confined to those who completed both the experimental (E) and placebo (P) phases of the study.
The distribution of the total plaque scores at the first examination is shown in Table 1 .

Table 2 shows the distribution of the two groups at the end of the first week of both the first and second phases of the study. The total scores on completion of three weeks in both phases of the study are shown in Table 3. The data were analyzed by multivariate analysis of variance. This was thought necessary because it did not seem appropriate to assume independence among observations made on the same subject. The total plaque score obviously did not have a normal distribution. However, the frequency tabulation shows that the distribution is reasonably symmetric, and the range of scores from 1 to 14 , together with the relatively large sample size, should insure the reliability of an analysis based on the assumption of multivariate normality.

The mean scores and standard deviations relative to the E-P sequence and the P-E sequence for the initial examination, the first week examination, and the third week examination are presented in Table 4 .

The mean plaque score of the initial experimental group decreased from 6.5 to 5.6, while the control 
TABLE 4

Mean plaque scores and standard deviations of all patients at the initial examination, the first week examination, and the third week examination.

\begin{tabular}{llcccccc}
\hline & & \multicolumn{2}{c}{ Initial Exam } & \multicolumn{2}{c}{ 1 Week Exam } & \multicolumn{2}{c}{3 Week Exam } \\
& & Mean & S.Dev. & Mean & S. Dev. & Mean & S. Dev. \\
\hline E-P Sequence & E & 6.5 & 2.4 & 5.8 & 2.5 & 5.6 & 2.7 \\
N = 45 & P & 6.4 & 2.4 & 5.8 & 2.7 & 6.3 & 2.9 \\
P-E Sequence & P & 6.3 & 2.1 & 5.7 & 2.3 & 5.9 & 2.8 \\
N = 46 & E & 5.9 & 2.1 & 5.7 & 2.2 & 5.2 & 2.2 \\
\hline
\end{tabular}

group score stayed almost constant from 6.4 to 6.3. In the second phase of the study, the experimental mean score dropped from 5.9 to 5.2 while the control group score dropped from 6.3 to only 5.9.

The analysis also shows that the residual effect, if any, from chewing the gum was eliminated by the six weeks interval between the two phases of the study. The score of the subjects who had started on the experimental gum returned to the original score before the second phase was started. The score of the subjects who started on the placebo was the same at the beginning of the second phase as it was at the end of the first phase.

The mean difference in total plaque scores at three weeks between the $\mathrm{E}$ group and $\mathrm{P}$ group is 0.3 , which is statistically significant at the 5 per cent level of probability (Table 5).

When the data for all subjects were plotted to determine any difference in linear trend, there was an initial drop in scores for both groups (Figure 1). This was interpreted as the Hawthorne Effect. However, after the first week, there was a continuing drop in the plaque score for the experimental group, whereas the placebo group tended to return to its original score. This was true whether the group started with the experimental chewing gum and went to placebo or started with the placebo and changed to the experimental. The difference in the linear trend has a significance level of .08. The difference is quite small, representing a decline of appröximately 0.2 of a score per week.

Many of the individuals had low plaque scores at the beginning of the investigation; thus there was little op-

TABLE 5

Statistical difference between the initial plaque scores and the final plaque scores. $\left(\mathrm{Eo}-\mathrm{E}_{3}\right)-\left(\mathrm{Po}-\mathrm{P}_{3}\right)$

\begin{tabular}{ccc}
\hline & Total Group & Initial Score $>6$ \\
\hline Difference E-P & 0.8 & 2.0 \\
S.E. & 0.5 & 0.5 \\
Difference P-E & 1.1 & 0.1 \\
S.E. & 0.5 & 0.5 \\
$\bar{X}$ Two Differences & 0.95 & 1.2 \\
S.E. & 0.33 & 0.4 \\
Stat. Sig. & $\mathrm{P}<.05$ & $\mathrm{P}<.02$ \\
\hline
\end{tabular}

portunity for the preparation to improve their score. All subjects having plaque scores less than six were omitted from the final analysis and the data reanalyzed. There were 28 subjects left in the E-P sequence and 31 in the P-E sequence. The linear trend of plaque status of subjects who had an initial plaque score of 6 or greater was analyzed (Figure 2). The statistical significance of plaque reduction when comparing the experimental to the placebo is at the 2 per cent level of probability (Table 5).

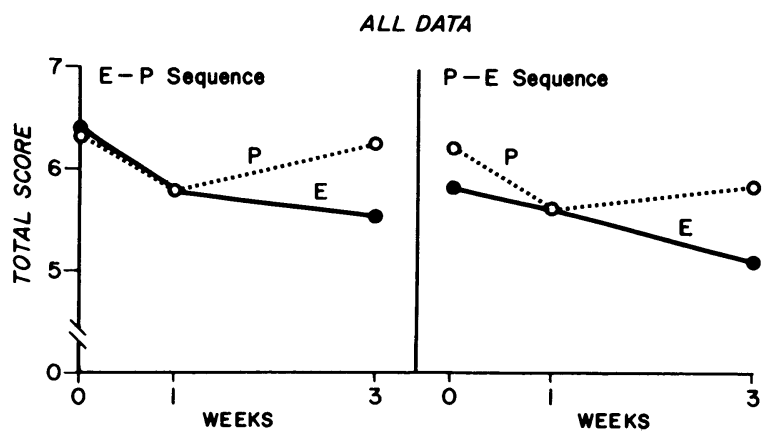

Figure 1. Linear graph of plaque scores of all subjects from 0 weeks to three weeks. E-P sequence (those patients who started on experimental gum and changed to placebo gum); $P$-E sequence (those subjects who started on placebo gum).

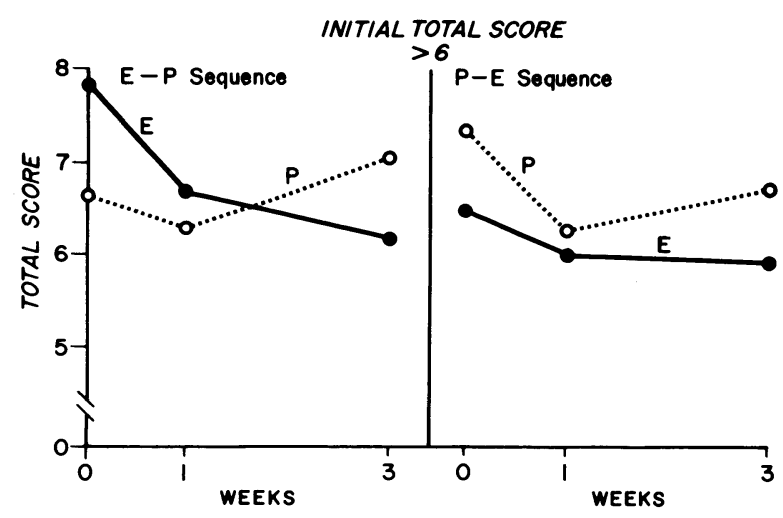

FIGURE 2. Linear graph of plaque scores for those subjects with an initial plaque score of greater than 6 . 

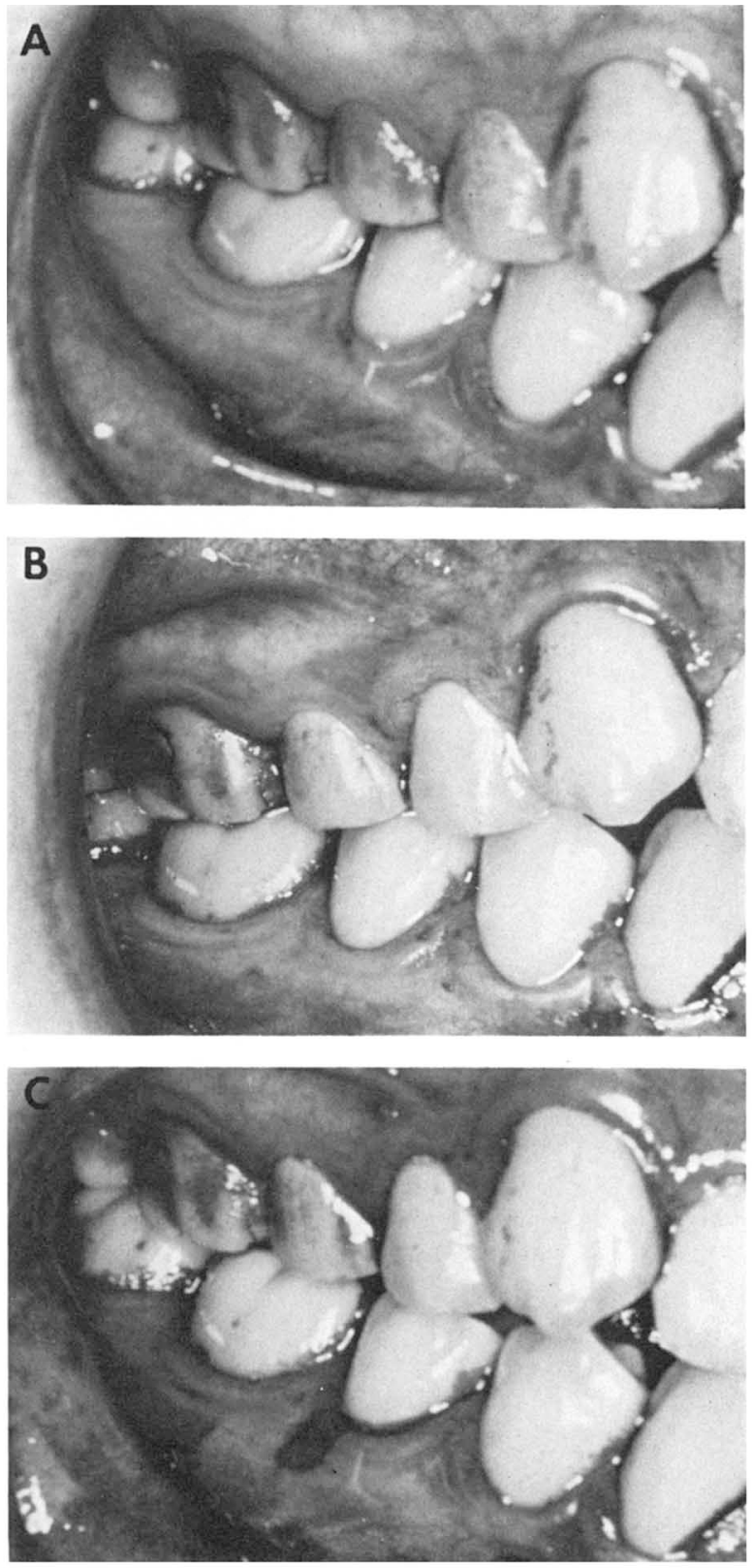

FIGURE 3. (A) Initial staining of bacterial plaque. (B) Distribution of stained plaque after using experimental gum for three weeks. (C) Return of stainable plaque after having used placebo gum for three weeks.

Figure 3a shows the initial staining of a subject. Reduction of stainable plaque after three weeks of using the experimental gum is evident in Figure 3b. Figure 3c shows a return of plaque to the original distribution after the placebo gum was used for three weeks. The before-and-after of another subject using the experimental gum is shown in Figure 4.

No changes were noted in the soft tissues of the oral cavity which could be directly attributed to the Viokasecontaining chewing gum. Several small ulcerative le- sions were observed that could be clinically diagnosed as recurrent aphthous stomatitis. These lesions occurred with equal frequency during the Viokase period and the placebo period.

Approximately 85 per cent of the subjects responded in a favorable manner to the placebo chewing gum. Only 45 per cent of the subjects had no complaints with the Viokase-containing gum. Of the 55 per cent who responded unfavorably, the most common complaints were irritation of the soft tissue of the oral cavity or an undesirable flavor. The consistency of the gum was also criticized. Irritation of the soft tissue was most frequently described as a burning sensation of the tongue when the gum was being chewed. The burning sensation frequently became less evident as the study progressed. There were no observable changes in the oral tissue that could explain the burning sensation of the tongue or other irritability in the oral cavity.

A one-week extension of the study was made for the group of subjects who had used Viokase for the first experimental period. Less than one-half of the total subjects took part in this portion of the study. It was an attempt to gain further information in regard to hypersensitivity or allergy. The comments received after this aspect of the study and the clinical examination were similar to those already described for the experimental group.
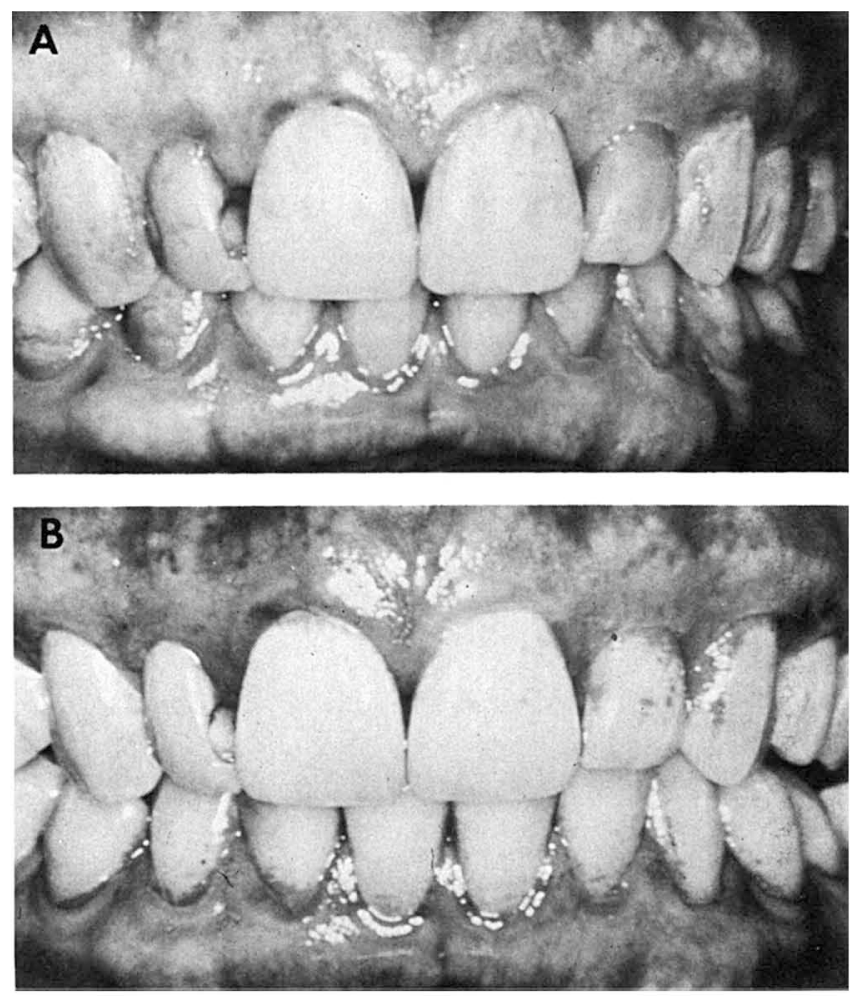

Figure 4. (A) Initial stainable plaque. (B) Stainable plaque after using experimental gum for three weeks. 
One patient developed aphthous stomatitis during the first experimental period and again during the one-week extension, when the Viokase-containing gum was resumed. The possibility of a hypersensitivity reaction in this one instance should be considered.

\section{Discussion}

Viokase is dehydrated and defatted pancreas. The pancreas is dried and defatted in a azeotrophic distillation that does not destroy the enzyme activity of whole pancreas. Viokase conforms to the National Formulary specifications for pancreatin. The concentration used in this study was one percent.

A review of the literature indicates that various enzyme preparations have been shown to be effective in bacterial plaque and calculus reduction. It is surprising that more emphasis has not been placed on studying them clinically. Perhaps the unfavorable response of subjects to the enzyme preparations is one of the primary reasons that more in-depth research has not been conducted.

It would seem ideal if a plaque-reducing, sugar-free chewing gum were available for use by the general population. However, when a high percentage of the subjects complain of irritation to the oral mucous membranes, the agent is probably inappropriate for general use. It is hoped that an acceptable form of the enzymes and vehicle for the active agent can be developed.

It was not determined from this study whether the low statistical significance in results with the experimental gum implies any clinical application in the reduction of periodontal disease. On the other hand, it must be realized that this was a short-term study demonstrating a beneficial trend of approximately an 0.2 plaque score unit reduction per week. If this reduction were to continue at the same rate, longer use of the agent might be clinically useful. If the teeth had been cleaned prior to the beginning of the study, the enzyme-containing gum might have been more effective in preventing plaque from occurring than it was in removing plaque that had already formed.

All subjects did not have the same response to the enzymes. Some did not reduce their plaque score, and a few even increased their plaque score while using the experimental gum. In a study of this nature, it is not possible to confirm that all subjects have followed instructions. Some of those who did not improve their plaque scores may not have used the gum as directed. On the other hand, failure to improve the scores may indicate that there is a difference in the substrate in plaque from one individual to another.

Further long-term studies using multiple enzymes need to be undertaken. Likewise, a vehicle which is more pleasant and less irritating to the subjects needs to be developed before this approach to plaque control can gain public acceptance.

\section{SUMMARY AND CONCLUSIONS}

A double-blind, cross-over experiment to determine the plaque removal potential of a Viokase-containing chewing gum was conducted on 91 subjects. Each phase of the investigation lasted three weeks. The following conclusions were drawn:

1. The experimental preparation containing Viokase produced a significantly greater plaque reduction than the placebo at the five per cent level of confidence. When a statistical analysis was performed on data of those subjects with an initial plaque score of six or greater, a somewhat greater level of confidence was shown at the two per cent level.

2. No changes were noted in the soft tissue examination that could be directly attributed to the Viokasecontaining chewing gum. Approximately 85 per cent of the subjects responded favorably to the placebo gum. Fifty-five per cent of the subjects responded unfavorably to the enzyme-containing gum. The most common complaints were irritation of the soft tissue of the oral cavity or an undesirable flavor. Irritation of the soft tissues was most frequently described as a burning sensation to the tongue when the gum was being chewed.

3. There were no allergic manifestations, except perhaps that one person developed aphthous stomatitis during the initial part of the study and also during the final part.

4. Long-term clinical studies of human subjects which measure plaque reduction and patient acceptance to various multiple enzyme preparations need to be conducted.

\section{REFERENCES}

1. Stewart, G. G.: Mucinase - A Possible Means of Reducing Calculus Formation. J. Periodontol., 23:85, 1952.

2. Aleece, A. A. and Forsher, B. J.: Calculus Reduction with a Mucinase Dentrifrice. J. Periodontol., 25:122, 1954.

3. Olinger, A. M.: Enzymatic Treatment of Periodontal Lesions. J. Amer. Dent. Assoc., 49:166, 1954.

4. Jensen, A. L.: Use of Dehydrated Pancreas in Oral Hygiene. J. Amer. Dent. Assoc., 59:923, 1959.

5. Ennever, J. and Sturzenberger, O. P.: Inhibition of Dental Calculus' Formation by Use of an Enzyme Chewing Gum. J. Periodontol., 32:331, 1961.

6. Packman, E. W., Abbott, D. D., Salisbury, G. B., and Harrison, J. W.: Effect of Enzyme-Chewing Gums upon Oral Hygiene. J. Periodontol., 34:255, 1963.

7. Harrison, J. W., Salisbury, G. B., Abbott, D. D., 
and Packman, E. W.: Effect of Enzyme-Tooth Paste upon Oral Hygiene. J. Periodontol., 34:334, 1963.

8. Fitzgerald, R. J., Keyes, P. H., Stoudt, T. H., and Spinell, D. M.: The Effects of a Dextranase Preparation on Plaque and Caries in Hamster, A Preliminary Report. J. Amer. Dent. Ass. 76:301, 1968.

9. Wasserman, B. H., Mandel, I. D., and Levy, B. M.: In vitro Calcification of Dental Calculus. J. Periodontol., 29:144, 1958.

10. Leung, S. W. and Draus, F. J.: Effect of Certain
Enzymes on Calculus Deposition. J. Dent. Res., 37:709, 1959 (Abstract No. 124).

11. Draus, F. J., Leung, S. W., and Miklos, F.: Toward a Chemical Inhibitor of Calculus. Dental Progress. 3:79, 1963.

12. Demmers, D. G. and Belting, C. M.: Effect of Surfactants and Proteolytic Enzymes on Artificial Calculus Formation. J. Periodontol., 38:294, 1967.

13. Greene, J. C. and Vermillion, J. R.: The Simplified Oral Hygiene Index. J. Amer. Dent. Assoc., 68:25, 1964.

\section{Abstracts}

\section{A Study of Bacteremia Following Wound Lavage}

Gross, A., Bhaskar, S. N., and Cutright, D. E. Oral Surg., 31:720, May, 1971

In two groups of rats the masseter muscles were cut $1.5 \mathrm{~cm}$ in length and to the mandibular ramus in depth. Bacteria were introduced into the wound (Staphylococcus aureus, Pseudomonas aeruginosa, Klebsiella pneumoniae, and Proteus mirabilis). Thirty minutes later a water lavage $\left(70 \mathrm{lb} . /\right.$ in..$\left.^{2}\right)$ was performed for 30 seconds. Blood samples were obtained by cardiac puncture $(5 \mathrm{ml}$.) at $2,5,10,20,30$ minutes and cultured. The second group of rats differed from the first only in that they had a $70 \%$ ethyl alcohol wipe prior to sampling. The results showed that except for occasional contamination, bacteremia was negligible in both groups. Department of Microbiology, U.S. Army Institute of Dental Research, Walter Reed Army Medical Center, Washington, D.C. 20012.

Effect of Pressure and Tip Modification On the Dispersion of Fluid Throughout Cells and Tissues DURING THE IRRIGATION OF EXPERIMENTAL WOUNDS

Carlson, H. C., Briggs, R. L., Green, V. A., and Stewart, J. L.

Oral Surg., 32:347, August, 1971

Lavage of 18 avulsive wounds in dogs with duplicate tip (single orifice, multijet, and radiant catheter tips) and pressure (30, 60, and 90 p.s.i.) variables was performed using pulsed lavage. An additional six wounds were irrigated with a rubber bulb syringe. The lavage solution was $100 \mathrm{ml}$. of a 25 percent aqueous solution of sodium diatrizoate, and the contaminant was iron filings. For another 24 animals, the lavage was $100 \mathrm{ml}$. of isotonic saline and there was no contaminant from iron filings. For another 24 animals, the lavage was $100 \mathrm{ml}$. of isotonic saline and there was no contaminant. Radiographs showed increasing dispersion of radiopaque fluid throughout the tissues as the pressure was increased with single orifice and radiant catheter tips. No spread occurred with the multijet tip or the bulb syringe. Microscopic study showed moderate fiber separation at 30 p.s.i. with the single orifice and radiant catheter tips, increasing at 60 and 90 p.s.i. The multijet tip produced no fiber separation at any of the pressures. The bulb syringe caused no fiber separation in $8 \times 5 \mathrm{~mm}$. wounds, but it did in $8 \times 20 \mathrm{~mm}$. wounds. It was concluded that the use of a multijet tip on a pulsed mechanical lavage device was the most favorable in terms of efficiency in removal of contaminant, combined with negligible tissue damage. Department of Research, University of Missouri, School of Dentistry, 650 E. 25th St., Kansas City, Mo. 64108 .

The Relationship Between Oral Symptoms, INSULIN RELEASE, aNd GluCOSE INTOLERANCE

Brody, H. A., Prendergast, J. J., and Silverman, S. Oral Surg., 31:777, June, 1971

Using 142 people who complained of oral symptoms of burning dryness, or gingival tenderness, comparisons were made between Glucose Tolerance Test findings, the magnitude and timing of the immunoreactive insulin release, and the effects of treatment with a new sulfonylurea. It was assumed that, at most, seven percent of the general population is affected by diabetes mellitus. In this study, 35 percent of the patient group with the oral symptoms had glucose tolerance curves indicative of diabetes. It was stated that oral symptoms may be helpful in monitoring the control of patients with diagnosed diabetes mellitus. Division of Oral Biology, University of California, San Francisco Medical Center, San Francisco, California 94122.

The Plaque Inhibiting Effect of Chlorhexidine-Containing Dentifrices

Gjermo, P., and Rölla, G.

Scand. J. Dent. Res., 79:126, No. 2, 1971

In a two-month double-blind study with 53 students using $0.8 \%$ and $0.6 \%$ chlorhexidine digluconate, the experimental groups had significantly $(\mathrm{p}<0.5)$ lower plaque scores than the placebo group. Storing the dentifrices at room temperature for up to six months resulted in no change of antibacterial activity as measured in vitro. Staining of silicate restorations was frequently noted, but no damage to oral mucosa or dorsum of the tongue was seen. Dept. of Periodontics, Dental Faculty, University of Oslo, Geitmyrsveien 69, Oslo, Norway. 